\title{
BOUNDED HOLOMORPHIC FUNCTIONS ON BOUNDED SYMMETRIC DOMAINS
}

\author{
JOEL M. COHEN AND FLAVIA COLONNA
}

\begin{abstract}
Let $D$ be a bounded homogeneous domain in $\mathbb{C}^{n}$, and let $\Delta$ denote the open unit disk. If $z \in D$ and $f: D \rightarrow \Delta$ is holomorphic, then $\beta_{f}(z)$ is defined as the maximum ratio $\left|\nabla_{z}(f) x\right| / H_{z}(x, \bar{x})^{1 / 2}$, where $x$ is a nonzero vector in $\mathbb{C}^{n}$ and $H_{z}$ is the Bergman metric on $D$. The number $\beta_{f}(z)$ represents the maximum dilation of $f$ at $z$. The set consisting of all $\beta_{f}(z)$, for $z \in D$ and $f: D \rightarrow \Delta$ holomorphic, is known to be bounded. We let $c_{D}$ be its least upper bound. In this work we calculate $c_{D}$ for all bounded symmetric domains having no exceptional factors and give indication on how to handle the general case. In addition we describe the extremal functions (that is, the holomorphic functions $f$ for which $\beta_{f}=c_{D}$ ) when $D$ contains $\Delta$ as a factor, and show that the class of extremal functions is very large when $\Delta$ is not a factor of $D$.
\end{abstract}

\section{INTRODUCTION}

A natural geometric question is to consider some class of smooth functions between Riemannian manifolds and ask under what conditions there is an upper bound on the amount of stretch possible for these functions. Under some conditions (e.g., analyticity, harmonicity) and for some manifolds, it may turn out that there is a bound which depends on the two manifolds. It is then natural to try to calculate the least upper bound and classify the extremal mappings.

In the most general setting, we can formalize these concepts as follows.

Definition 1. Let $M$ and $N$ be Riemannian manifolds and $f: M \rightarrow N$ a smooth function. Then $f$ induces a map of the tangent bundles, $d f: T M \rightarrow$ $T N$. At each point $x \in M, d f_{x}: T_{x} M \rightarrow T_{f(x)} N$ is a linear transformation of Euclidean spaces. Define $\beta_{f}(x)$ as the norm of $d f_{x}$ considered as map of normed vector spaces. We call $f$ Bloch if the constant $\beta_{f}=\sup _{x \in M} \beta_{f}(x)$ is finite. The number $\beta_{f}$ is called the Bloch constant of $f$.

It is easy to see that a smooth Lipschitz function (i.e., a function with bounded dilation) is necessarily Bloch. In fact given $x \in M$, if $\lambda$ is a positive constant such that $d_{N}(f(x), f(y)) \leq \lambda d_{M}(x, y)$, for every $y \in M$, then $\beta_{f}(x) \leq \lambda$. Here $d_{M}$ and $d_{N}$ denote the distance functions induced by the Riemannian metrics of $M$ and $N$, respectively.

Received by the editors May 6, 1992.

1980 Mathematics Subject Classification (1985 Revision). Primary 32M15; Secondary 32A10, $30 \mathrm{D} 14$.

Key words and phrases. Bloch, bounded symmetric domains, Lipschitz. 
On the other hand, if $f$ is a Bloch function, then the length of the image of any curve in $M$ is no greater than the length of the original curve multiplied by the Bloch constant of $f$. For if $\gamma$ is a linear approximation of length $l$ to a curve through $x \in M$, then its image $d f_{x}(\gamma)$ will have length no greater than $\beta_{f}(x) l$. Now any two points $x, y \in M$ can be connected by curves whose lengths approach $d_{M}(x, y)$. Thus $d_{N}(f(x), f(y)) \leq \beta_{f} d_{M}(x, y)$, so that $f$ is a Lipschitz function with Lipschitz number $\beta_{f}$.

In [H1] Hahn introduced the notion of Bloch function on a bounded homogeneous domain in $\mathbb{C}^{n}$, with $n \in \mathbb{N}$. We recall that a domain $D \in \mathbb{C}^{n}$ is said to be homogeneous if the group of biholomorphic transformations of $D$ onto itself acts transitively on $D$. In this case, one takes the Riemannian metric induced by the Bergman metric (cf. [H3]). The Bergman metric is the natural metric on such domains as it is preserved under all biholomorphic transformations. Timoney studied extensively the properties of Bloch functions from a bounded homogeneous domain into the complex plane (cf. [T1, T2]). Both Timoney's and Hahn's definitions are subsumed in the above definition, by taking the Bergman metric on the domain and the Euclidean metric on $\mathbb{C}$. In this paper we shall conform to the approach and terminology used by Timoney. For the cases we study here we use the following.

Definition 2. Let $D$ be a bounded homogeneous domain in $\mathbb{C}^{n}$. A holomorphic function $f: D \rightarrow \mathbb{C}$ is called Bloch if $\sup _{z \in D} \beta_{f}(z)$ is finite, where

$$
\beta_{f}(z)=\sup \left\{\frac{\left|\nabla_{z}(f) x\right|}{\left[H_{z}(x, \bar{x})\right]^{1 / 2}}: \mathbf{0} \neq x \in \mathbb{C}^{n}\right\},
$$

with $\nabla_{z}(f) x=\sum_{j=1}^{n}\left(\partial f / \partial z_{j}\right)(z) x_{j}$ and where $H_{z}(u, \bar{v})$ is the Bergman metric on $D$. The quantity $\beta_{f}=\sup _{z \in D} \beta_{f}(z)$ is the Bloch constant of $f$.

If $z \in D$, a domain in $\mathbb{C}^{n}$, then $T_{z} D$ is naturally identifiable with $\mathbb{C}^{n}$, but the inner product on it is given by $H_{z}(u, \bar{v})$ instead of the standard inner product on $\mathbb{C}^{n}$. The corresponding Riemannian metric is given by $g_{z}(u, v)=$ $\operatorname{Re} H_{z}(u, \bar{v})$.

Timoney showed that as in the case of the open unit disk the set $\mathscr{B}(D)$ of all Bloch functions on a bounded homogeneous domain $D$ modulo the constants constitutes a complex Banach space with norm $\|f\|_{\mathscr{B}(D)}=\beta_{f}$. He then proved that every bounded holomorphic function $f: D \rightarrow \mathbb{C}$ is necessarily a Bloch function and $\|f\|_{\mathscr{B}(D)} \leq c\|f\|_{\infty}$, where $c$ is a constant depending on $D$, and \|\|$_{\infty}$ is the supremum norm. In the cases of the unit ball $B_{n}=\left\{z \in \mathbb{C}^{n}:\|z\|<\right.$ $1\}$ and the polydisk $\Delta^{n}=\left\{\left(z_{1}, \ldots, z_{n}\right) \in \mathbb{C}^{n}:\left|z_{j}\right|<1, j=1, \ldots, n\right\}$ he took $c$ equal to $\sqrt{2} /(B \sqrt{n+1})$ and $1 / B$, respectively, (cf. [T1, p. 260]) where $B$ is a positive constant known as the Bloch number.

Let $C_{D}$ be the smallest possible value of $c$ in the above inequality. We shall refer to $c_{D}$ as the Bloch constant of $D$. Without loss of generality we may normalize by considering functions from $D$ to $\Delta$, so that

$$
\begin{aligned}
c_{D} & =\sup \left\{\beta_{f} \mid f: D \rightarrow \Delta \text { holomorphic }\right\} \\
& =\sup \left\{\beta_{f}(z) \mid f: D \rightarrow \Delta \text { holomorphic, } z \in D\right\} .
\end{aligned}
$$


For $n=1$, where $D=B_{1}=\Delta^{1}=\Delta$, the constant $c_{D}$ is equal to 1 by the Schwarz-Pick Lemma, which states that for any analytic function $f: \Delta \rightarrow \Delta$, we have that $\left(1-|z|^{2}\right)\left|f^{\prime}(z)\right| \leq 1-|f(z)|^{2}$ for all $z \in \Delta$, with equality holding at any point if and only if $f$ is an automorphism. The term $1 / B$, which is between 2.11 and 2.31 (cf. [H2]), is, therefore, unnecessary. Thus two interesting natural problems arise for $B_{n}$ and $\Delta^{n}$, or more generally, for any bounded homogeneous domain $D$ in $\mathbb{C}^{n}$.

(1) Determine $c_{D}$.

(2) Classify the extremal functions, that is, those holomorphic functions $f: D \rightarrow \Delta$ such that $\beta_{f}=c_{D}$.

In [C2], the analytic functions mapping $\Delta$ into itself were studied, and the extremal functions were classified. In [C3], harmonic self-mappings of $\Delta$ were studied, the corresponding $c_{\Delta}$ was found to be $4 / \pi$, and the extremal functions were described (but not as precisely as in the analytic case).

In this paper we shall study these problems for the case of $D$ a bounded symmetric domain in $\mathbb{C}^{n}$, and for holomorphic functions from $D$ into $\Delta$. It is well known that these domains are homogeneous (cf. [H3, p. 301]). For the classical domains (those which do not contain an exceptional factor (see $\S 2$ ) we shall answer question (1) completely (Theorem 3 ). We shall describe an upper bound for the general case (Theorem 1).

In $\S 3$ we shall present some variations of the Schwarz Lemma relevant to the study of the extremal functions.

Question (2) divides into two cases: (i) $\beta_{f}(w)<c_{D}$, for all $w \in D$, or (ii) $\beta_{f}(w)=c_{D}$, for some $w \in D$.

Theorem 7 shows how to relate case (i) to case (ii), although a lot of information may be lost in the process. It seems unlikely that we can get same precision as in [C2]. For the case (ii), we are able to obtain much more information (Theorem 6). If in addition the unit disk is a factor of $D$, we are able to give a complete description of the space of the extremal functions of this type (it is a real three-dimensional manifold) (Corollary 3). But for all the other classical cases, we show (Theorem 8) that the class of such functions is large enough to contain an infinite direct product of copies of the space of holomorphic functions from $D$ to $\Delta$ !

We shall first obtain estimates on $c_{D}$, which depend on the irreducible factors of $D$ and the location of the boundary points of $D$ closest to the origin in a sense related to the Bergman metric of $D$. As a consequence, for example, we find that $c_{D}$ is $\sqrt{2 /(n+1)}$ if $D$ is the $n$-ball and 1 if $D$ is a polydisk. (Thus the factor $1 / B$ is not necessary in any dimension.)

Another interpretation of $c_{D}$ involves the Carathéodory distance $d_{C}$ on the domain $D$. Recall that if $z, w \in D$, then $d_{C}(z, w)=\sup _{f} d_{\Delta}(f(z), f(w))$, where the supremum is taken over all holomorphic functions $f: D \rightarrow \Delta$, and $d_{\Delta}$ is the hyperbolic distance on $\Delta$. It is easy to see that

$$
c_{D}=\sup _{z \neq w \in D} \frac{d_{C}(z, w)}{d_{D}(z, w)},
$$

where $d_{D}$ is the distance induced by the Bergman metric on $D$.

The authors wish to thank William Goldman and David Singman for many useful and productive discussions. 


\section{BLOCH CONSTANT OF A BOUNDED SYMMETRIC DOMAIN}

We recall that a domain $D$ in $\mathbb{C}^{n}$ is called symmetric if for every point $z_{0} \in D$ there exists an automorphism $S$ of $D$ which is an involution (i.e., $S^{2}$ is the identity) and for which $z_{0}$ is an isolated fixed point. By an automorphism of $D$ we mean a biholomorphic function of $D$ onto itself.

Remark 1. Let $D$ be any bounded symmetric domain, $f: D \rightarrow \Delta$ a holomorphic function and $S$ an automorphism of $D$. Fix a point $z_{0} \in D$. For any $z \in D$ there is an automorphism $S$ of $D$ such that $S\left(z_{0}\right)=z$, by the homogeneity of $D$. Since $S$ preserves the Bergman metric, we get that $\beta_{f}(z)=\beta_{f \circ S}\left(z_{0}\right)$. Thus $c_{D}=\sup \left\{\beta_{f}\left(z_{0}\right) \mid f: D \rightarrow \Delta\right.$ holomorphic $\}$. Observe that $c_{D}$ is invariant under biholomorphic transformations.

Let $D$ be a bounded symmetric domain. By a result due to Cartan [C1], $D$ is biholomorphically equivalent to a finite product of irreducible bounded symmetric domains, unique up to order. He furthermore classified all the irreducible domains into six classes, the first four of which form infinite families, and are the Cartan classical domains of types I, II, III, and IV. The remaining two classes each contain a single domain, the exceptional domains $E_{\mathrm{III}}$ and $E_{\mathrm{VII}}$, which are more complicated to describe.

In this section we shall calculate precisely $c_{D}$ in the case when $D$ does not contain any exceptional factors. In the general case we shall get an upper bound on $c_{D}$.

Notation. Let $\mathscr{K}_{m \times n}(R)$ denote the set of $(m \times n)$-matrices over a ring $R$, possibly nonassociative. Let $\mathscr{M}_{n}(R)$ be the set of $(n \times n)$-matrices over $R$. If $R$ does not appear it is assumed to be $\mathbb{C}$. Let $\mathbf{K}$ denote the (nonassociative) ring of the Cayley numbers over $\mathbb{C}$ (cf. Appendix).

The Cartan classical domains and their corresponding Bergman metrics are described as follows:

$$
\begin{aligned}
& \text { Type } \mathrm{I}_{m, n}:\left\{Z \in \mathscr{M}_{m \times n} \mid \mathrm{I}_{m}-Z Z^{*}>0\right\}, \quad \text { for } m \geq n \geq 1 \text {, } \\
& H_{Z}(U, \bar{V})=\frac{m+n}{2} \operatorname{Trace}\left[\left(\mathrm{I}_{m}-Z Z^{*}\right)^{-1} U\left(\mathrm{I}_{n}-Z^{*} Z\right)^{-1} V^{*}\right] \text {, } \\
& \text { Type } \mathrm{II}_{n}:\left\{Z \in \mathscr{M}_{n} \mid Z \text { symmetric, } \mathrm{I}_{n}-Z Z^{*}>0\right\}, \quad \text { for } n \geq 1 \text {, } \\
& H_{Z}(U, \bar{V})=\frac{n+1}{2} \operatorname{Trace}\left[\left(\mathrm{I}_{n}-Z Z^{*}\right)^{-1} U\left(\mathrm{I}_{n}-Z^{*} Z\right)^{-1} V^{*}\right] \text {, } \\
& \text { Type } \mathrm{III}_{n}:\left\{Z \in \mathscr{K}_{n} \mid Z \text { skew-symmetric, } \mathrm{I}_{n}-Z Z^{*}>0\right\}, \quad \text { for } n \geq 2 \text {, } \\
& H_{Z}(U, \bar{V})=\frac{n-1}{2} \operatorname{Trace}\left[\left(\mathrm{I}_{n}-Z Z^{*}\right)^{-1} U\left(\mathrm{I}_{n}-Z^{*} Z\right)^{-1} V^{*}\right] \text {, } \\
& \text { Type } \operatorname{IV}_{n}:\left\{z=\left(z_{1}, \ldots, z_{n}\right) \in \mathbb{C}^{n}:\left|\sum_{j=1}^{n} z_{j}^{2}\right|^{2}+1-2 \sum_{j=1}^{n}\left|z_{j}\right|^{2}>0\right. \text {, } \\
& \left.\left|\sum_{j=1}^{n} z_{j}^{2}\right|<1\right\} \\
& \text { for } n \geq 1, n \neq 2 \text {, } \\
& H_{z}(u, \bar{v})=n A u\left[A\left(\mathrm{I}_{n}-z^{T} \bar{z}\right)+\left(\mathrm{I}_{n}-z^{T} \bar{z}\right) z^{*} z\left(\mathrm{I}_{n}-z^{T} \bar{z}\right)\right] v^{*},
\end{aligned}
$$


where $\mathrm{I}_{n}$ denotes the identity matrix of order $n, Z^{*}$ is the complex conjugate of the transpose of $Z, z^{T}$ is the transpose of the vector $z$, and $A=$ $\left|\sum_{j=1}^{n} z_{j}^{2}\right|^{2}+1-2 \sum_{j=1}^{n}\left|z_{j}\right|^{2}$.

Here we use Kobayashi's definitions and notations (cf. [K1, p. 34]), except that the Bergman metrics have been divided by 4 . The case $n=2$ cannot occur for a domain of type $\mathrm{IV}_{n}$, since it turns out to be reducible (see the observation following Lemma 3 ).

The unit disk can be described as a domain of type $\mathrm{I}_{1,1}$, type $\mathrm{II}_{1}$, type $\mathrm{III}_{2}$, and type $\mathrm{IV}_{1}$. We also have the following isomorphisms: $\mathrm{III}_{3} \cong \mathrm{I}_{3,1}, \mathrm{IV}_{3} \cong$ $\mathrm{II}_{2}, \mathrm{IV}_{4} \cong \mathrm{I}_{2,2}$, and $\mathrm{IV}_{6} \cong \mathrm{III}_{4}$. (Cf. [S1].) So we could achieve uniqueness in the above list by considering the following dimensional restrictions on Cartan classical domains: type $\mathrm{II}_{n}$, for $n \geq 2$, type $\mathrm{III}_{n}$, for $n \geq 5$, type $\mathrm{IV}_{n}$, for $n \geq 5$.

A description of the exceptional bounded symmetric domains can be found in [I1, K2, D1]. Drucker [D1] proved that all these descriptions are of the same spaces. There is also a brief discussion of these domains in the Appendix.

Given a bounded symmetric domain, we will say that it is in standard form if it is written as $D=D_{1} \times D_{2} \times \cdots \times D_{t}$, where each $D_{j}$ is one of the Cartan classical domains listed above or one of the exceptional domains as described in the Appendix. The latter are given as domains $E_{\mathrm{III}}$ of $\mathbf{K}^{2}$ and $E_{\mathrm{VII}}$ of $\mathbb{C}^{3} \oplus \mathbf{K}^{3}$. If a bounded symmetric domain is biholomorphically equivalent to one in standard form with no exceptional factors, we shall refer to it as a classical domain.

Remark 2. All of the Cartan classical domains, as well as the two exceptional domains, are balanced; that is, they satisfy the property $\bar{\Delta} D=D$. (Since they are bounded, they also satisfy $\Delta \partial D=D$.) This is easy to see for all the classical domains, except for those of type IV, since each is defined in terms of quadratic inequalities. The verification that the exceptional cases are also defined in terms of quadratic inequalities requires going through each step of the definitions given in the Appendix, but it can be seen to hold as well.

Assume now that $D$ is of type IV. Then the second inequality defining $D$ is quadratic. To check that the first inequality remains true when $z$ is replaced by $\alpha z$ for $\alpha \in \bar{\Delta}$, we let $r=|\alpha|^{2}$. We need to verify that

$$
r^{2}\left|\sum_{j=1}^{n} z_{j}^{2}\right|^{2}+1-2 r \sum_{j=1}^{n}\left|z_{j}\right|^{2}>0, \quad \text { for } r \in[0,1] .
$$

This inequality is clearly true for $r=0,1$, and thus if it fails for any $r \in(0,1)$, both roots of the left-hand side must be in $(0,1)$. Since except for $z=0$ we have

$$
\left|\sum_{j=1}^{n} z_{j}^{2}\right|^{2}<\left|\sum_{j=1}^{n} z_{j}^{2}\right| \leq \sum_{j=1}^{n}\left|z_{j}\right|^{2},
$$

one sees that this is impossible.

Let $D$ be any bounded symmetric domain. Then $D$ is biholomorphically equivalent to some bounded symmetric domain $D^{\prime}$ in standard form, unique 
up to the ordering of the irreducible factors. Define the inner radius of $D$ by

$$
r_{D}=\inf _{x \in \partial D^{\prime}} H_{0}(x, \bar{x})^{1 / 2} .
$$

Since $\partial D^{\prime}$ is compact, this infimum is actually a minimum.

We now calculate $r_{D}$.

Proposition 1. Let $D$ be a Cartan classical domain. Then

$$
r_{D}= \begin{cases}\sqrt{(m+n) / 2}, & \text { if } D \text { is of type } \mathrm{I}_{m, n}, \\ \sqrt{(n+1) / 2}, & \text { if } D \text { is of type } \mathrm{II}_{n}, \\ \sqrt{n-1}, & \text { if } D \text { is of type } \mathrm{III}_{n}, \\ \sqrt{n / 2}, & \text { if } D \text { is of type } \mathrm{IV}_{n} .\end{cases}
$$

Proof. In the cases when $D$ is of type $\mathrm{I}_{m, n}, \mathrm{II}_{n}, \mathrm{III}_{n}$, the elements of $D$ are matrices in $\mathscr{K}_{m \times n}, \mathscr{M}_{n}, \mathscr{M}_{n}$, respectively. But as a domain, $D$ is inside $\mathbb{C}^{t}$, where $t=m n, n(n+1) / 2, n(n-1) / 2$, respectively. Beside the ordinary matrix product-which we write in the usual way-we sometimes need to look at the $\mathbb{C}$-bilinear scalar product of two elements considered as vectors in $\mathbb{C}^{t}$. We shall use the symbol $*$ to represent this product. For example if $D$ is of type $\mathrm{II}_{2}$, the element $\left(\begin{array}{ll}a & b \\ b & c\end{array}\right)$ may also be thought of as

$$
\left(\begin{array}{l}
a \\
b \\
c
\end{array}\right) \text { or as }\left(\begin{array}{lll}
a & b & c
\end{array}\right)
$$

as elements of $\mathbb{C}^{3}$. So

$$
\left(\begin{array}{ll}
a & b \\
b & c
\end{array}\right) *\left(\begin{array}{ll}
d & e \\
e & f
\end{array}\right)=\left(\begin{array}{lll}
a & b & c
\end{array}\right)\left(\begin{array}{l}
d \\
e \\
f
\end{array}\right)=a d+b e+c f .
$$

We shall also, consistent with the notation used in Kobayashi [K1], write elements of the tangent space as matrices in $\mathscr{M}_{m \times n}, \mathscr{M}_{n}, \mathscr{M}_{n}$, respectively, which satisfy no condition, symmetry, skew-symmetry, respectively. In the same way, if $f$ is a function on $D$, we shall write $\nabla f$ as a matrix of the same type, repeating, where necessary, the partial derivatives with respect to the variables that are dependent: in case $\mathrm{II}_{n}, z_{i j}=z_{j i}$; in case $\mathrm{III}_{n}, z_{i j}=-z_{j i}$ and $z_{i i}=0$. With this notation, we have

$$
\beta_{f}(W)=\sup _{X \neq O} \frac{\left|\nabla_{W}(f) * X\right|}{H_{W}(X, \bar{X})^{1 / 2}} .
$$

We shall first consider the case when $D$ is of type $\mathrm{I}_{m, n}$, or $\mathrm{II}_{n}$. Set

$$
\Xi=\left(\begin{array}{cccc}
1 & 0 & \cdots & 0 \\
0 & 0 & \cdots & 0 \\
\vdots & \vdots & \ddots & \vdots \\
0 & 0 & \cdots & 0
\end{array}\right)
$$

Then $\Xi \in \partial D$, and $H_{0}(\Xi, \bar{\Xi})=\varepsilon \operatorname{Trace}\left(\Xi^{*}\right)=\varepsilon$, where $\varepsilon=(m+n) / 2$ if $D$ is of type $\mathrm{I}_{m, n}$ and $\varepsilon=(n+1) / 2$ if $D$ is of type $\mathrm{II}_{n}$. Furthermore, if $X \in \partial D$, then $X X^{*} \leq \mathrm{I}$ but $X X^{*} \nless \mathrm{I}$, where $\mathrm{I}$ denotes the identity matrix. 
This means that $X X^{*}$ has 1 as an eigenvalue. Since all eigenvalues of $X X^{*}$ are nonnegative real numbers, the trace of $X X^{*}$ is necessarily at least 1 . Therefore

$$
\min _{X \in \partial D} H_{0}(X, \bar{X})=\varepsilon=H_{0}(\Xi, \bar{\Xi}) .
$$

This proves parts (1) and (2).

Now let $D$ be of type III $_{n}$. Set

$$
\Xi=\left(\begin{array}{cccc}
0 & 1 & \cdots & 0 \\
-1 & 0 & \cdots & 0 \\
\vdots & \vdots & \ddots & \vdots \\
0 & 0 & \cdots & 0
\end{array}\right)
$$

Then $\Xi \in \partial D$, and $H_{0}(\Xi, \bar{\Xi})=\varepsilon \operatorname{Trace}\left(\Xi \Xi^{*}\right)=2 \varepsilon$, where $\varepsilon=(n-1) / 2$. Furthermore, if $X \in \partial D$, then as in the previous cases $X X^{*}$ has 1 as an eigenvalue. Since all eigenvalues of $X X^{*}$ are nonnegative real numbers, the trace of $X X^{*}$ is necessarily at least 2 by Lemma 1 which follows. Therefore

$$
\min _{X \in \partial D} H_{0}(X, \bar{X})=H_{0}(\Xi, \bar{\Xi})=n-1 .
$$

Lemma 1. Let $A$ be a skew-symmetric matrix. Let $\lambda$ be a positive eigenvalue of $A A^{*}$. Then $\lambda$ has multiplicity at least two. In particular, $\operatorname{Trace}\left(A A^{*}\right) \geq 2 \lambda$. Proof. By hypothesis $A^{T}=-A$. Thus $A^{*}=-\bar{A}$, and so $A A^{*}$ and $A^{*} A$ are complex conjugates to one another. Assume that $A A^{*} v=\lambda v$, where $v$ is a nonzero vector. Taking the conjugate of this equality, we get that $A^{*} A \bar{v}=\lambda \bar{v}$. Thus $A A^{*}(A \bar{v})=A \lambda \bar{v}=\lambda(A \bar{v})$. Note that $A \bar{v}$ is nonzero, since $A^{*}(A \bar{v})=$ $\lambda \bar{v} \neq 0$. Thus $v$ and $A \bar{v}$ are eigenvectors of $A A^{*}$ for the eigenvalue $\lambda$. So the multiplicity of $\lambda$ is at least two unless $A \bar{v}=\mu v$ for some $\mu \in \mathbb{C}, \mu \neq 0$. But if we were to assume this last equality, we would also have that $\bar{A} v=\overline{\mu v}$. Then $A^{*} A \bar{v}=-\bar{A} A \bar{v}=-\bar{A} \mu v=-\mu \bar{A} v=-|\mu|^{2} \bar{v}$. So $-|\mu|^{2}$ would be a negative eigenvalue of $A^{*} A$, a positive definite matrix. This yields a contradiction.

Noting that the trace of $A A^{*}$ is the sum of its eigenvalues-all of which are nonnegative-counted according to their multiplicities, the final result follows.

To complete the proof of Proposition 1, we now assume that $D$ is of type $\mathrm{IV}_{n}$. So

$$
D=\left\{\left(x_{1}, \ldots, x_{n}\right) \in \mathbb{C}^{n}: \sum_{j=1}^{n}\left|x_{j}\right|^{2}<\frac{1}{2}\left(1+\left|\sum_{j=1}^{n} x_{j}^{2}\right|^{2}\right) \text { and }\left|\sum_{j=1}^{n} x_{j}^{2}\right|<1\right\} \text {. }
$$

Note that the condition $\left|\sum_{j=1}^{n} x_{j}^{2}\right|=1$ implies that

$$
\sum_{j=1}^{n}\left|x_{j}\right|^{2} \geq 1=\frac{1}{2}\left(1+\left|\sum_{j=1}^{n} x_{j}^{2}\right|^{2}\right) \text {. }
$$

Thus $x=\left(x_{1}, \ldots, x_{n}\right) \in \partial D$ if and only if

$$
\sum_{j=1}^{n}\left|x_{j}\right|^{2}=\frac{1}{2}\left(1+\left|\sum_{j=1}^{n} x_{j}^{2}\right|\right)
$$


In particular

$$
H_{0}(x, \bar{x})=n \sum_{j=1}^{n}\left|x_{j}\right|^{2} \geq \frac{n}{2} \quad \text { if } x \in \partial D .
$$

Thus the point $\xi=\left(\frac{1}{2},-\frac{i}{2}, 0, \ldots, 0\right)$ belongs to $\partial D$ and satisfies the conditions $H_{0}(\xi, \bar{\xi})=n / 2=\min _{x \in \partial D} H_{0}(x, \bar{x})$. This completes the proof of Proposition 1.

Theorem 1. Let $D$ be a bounded symmetric domain in $\mathbb{C}^{n}$. Then $c_{D} \leq 1 / r_{D}$.

Proof. Without loss of generality, we may assume that $D$ is in standard form. If $f: D \rightarrow \Delta$ is holomorphic, then

$$
\beta_{f}(0)=\sup _{x \in \partial D} \frac{\left|\nabla_{0}(f) x\right|}{\left[H_{0}(x, \bar{x})\right]^{1 / 2}} .
$$

In order to see this, we recall that in Definition 2 the supremum defining $\beta_{f}(z)$ is taken over all nonzero $x \in \mathbb{C}^{n}$. Observe that multiplying $x$ by any nonzero complex number leaves the quotient unchanged. In particular, if $D$ is a bounded symmetric domain in standard form, then $D$ contains a neighborhood of zero in $\mathbb{C}^{n}$. So for each nonzero $x \in \mathbb{C}^{n}$ we can find $\lambda \in \mathbb{C}$ such that $\lambda x \in \partial D$. Thus without loss of generality, we can define $\beta_{f}(z)$ by taking the supremum over all $x \in \partial D$. Of course, this supremum is a maximum, by the compactness of $\partial D$.

Fix $x \in \partial D$. By Remark 2 we may define a function $g: \Delta \rightarrow \Delta$ by $g(z)=$ $f(z x)$. Then $g^{\prime}(z)=\nabla_{z x}(f) x$. So $\left|\nabla_{0}(f) x\right|=\left|g^{\prime}(0)\right| \leq 1$, by the SchwarzPick Lemma. Hence

$$
\beta_{f}(0) \leq \sup _{x \in \partial D} \frac{1}{H_{0}(x, \bar{x})^{1 / 2}}=\frac{1}{\inf _{x \in \partial D} H_{0}(x, \bar{x})^{1 / 2}}=\frac{1}{r_{D}} .
$$

By Remark 1 it follows that $c_{D} \leq 1 / r_{D}$.

We now show that, in the case when $D$ has only Cartan classical (irreducible) factors, the inequality in the statement of Theorem 1 is an actual equality. We accomplish this by producing a function $f: D \rightarrow \Delta$ and a point $\xi \in \partial D$ such that $\left|\nabla_{0}(f) \xi\right|=1$, and the greatest lower bound for $H_{0}(x, \bar{x})$ is attained at $\xi$.

We begin by studying the case when $D$ is a Cartan classical domain.

Theorem 2. Let $D$ be a Cartan classical domain. Then there exists a holomorphic function $f: D \rightarrow \Delta$ and a point $\xi \in \partial D$ such that $\left|\nabla_{0}(f) \xi\right|=1$ and $\inf _{x \in \partial D} H_{0}(x, \bar{x})=H_{0}(\xi, \bar{\xi})$. In particular, $c_{D}=1 / r_{D}$.

For the proof we need the following elementary lemmas.

Lemma 2. Let $Z$ be a matrix satisfying $Z Z^{*}<\mathrm{I}$, where I denotes the identity matrix. If $z$ is one of the rows of $Z$, then $\|z\|<1$.

Proof. Let $n$ be the order of the matrix $Z Z^{*}$. Then for any vector $v$ in $\mathbb{C}^{n}$, we have $\left\|Z^{*} v\right\|^{2}=Z^{*} v \cdot Z^{*} v=Z Z^{*} v \cdot v<v \cdot v=\|v\|^{2}$. Let $z$ be the $k$ th row of $Z$. Taking $v$ equal to the $k$ th element of the standard basis of $\mathbb{C}^{n}$, the vector $z^{*}=Z^{*} v$ is the $k$ th column of $Z^{*}$, which is the adjoint of $z$. So $\|z\|=\left\|z^{*}\right\|=\left\|Z^{*} v\right\|<\|v\|=1$.

We now gather together some useful results concerning the domains of type IV. 
Lemma 3. Let $D_{k}$ be a bounded symmetric domain of type $\mathrm{IV}_{k}$.

(a) If $\left(z_{1}, z_{2}, \ldots, z_{k}\right) \in D_{k}$ then for $m<k$, we have $\left(z_{1}, z_{2}, \ldots, z_{m}\right) \in$ $D_{m}$.

(b) If $z_{1}, z_{2} \in \mathbb{C}$, then $\left(z_{1}, z_{2}\right) \in D_{2}$ if and only if $\left|z_{1}+i z_{2}\right|<1$ and $\left|z_{1}-i z_{2}\right|<1$.

(c) If $\left(z_{1}, z_{2}, z_{3}\right) \in D_{3}$ then

$$
\left|z_{1} \pm i z_{2}\right|^{2}+\left|z_{3}\right|^{2}<1
$$

Lemma 3(b) shows why we cannot allow $n=2$ in the classification of the type $\mathrm{IV}_{n}$ spaces. Such a space would be biholomorphically equivalent to the polydisk $\Delta^{2}$.

Proof. We begin by showing that

$$
\left(z_{1}, z_{2}, \ldots, z_{n}\right) \in D_{n} \Rightarrow\left(z_{1}, z_{2}, \ldots, z_{n-1}\right) \in D_{n-1} \text {. }
$$

Recalling that $\left(z_{1}, z_{2}, \ldots, z_{n}\right) \in D_{n}$ if and only if

$$
\left|\sum_{j=1}^{n} z_{j}^{2}\right|^{2}+1-2 \sum_{j=1}^{n}\left|z_{j}\right|^{2}>0
$$

and

$$
\left|\sum_{j=1}^{n} z_{j}^{2}\right|<1
$$

we may express membership in $D_{n}$ by the double inequality

$$
\sum_{j=1}^{n}\left|z_{j}\right|^{2}<\frac{1}{2}\left(1+\left|\sum_{j=1}^{n} z_{j}^{2}\right|^{2}\right)<1
$$

We must show that if (5) holds then

$$
\sum_{j=1}^{n-1}\left|z_{j}\right|^{2}<\frac{1}{2}\left(1+\left|\sum_{j=1}^{n-1} z_{j}^{2}\right|^{2}\right)<1 .
$$

Observe that the second inequality of (6) holds since

$$
\left|\sum_{j=1}^{n-1} z_{j}^{2}\right| \leq \sum_{j=1}^{n-1}\left|z_{j}\right|^{2} \leq \sum_{j=1}^{n}\left|z_{j}\right|^{2}<1
$$

by (5). Now let us verify the first inequality of (6).

First note that since

$$
\left|\sum_{j=1}^{n-1} z_{j}^{2}\right| \leq \sum_{j=1}^{n-1}\left|z_{j}\right|^{2}<1-\left|z_{n}\right|^{2} \leq 1-\frac{1}{2}\left|z_{n}\right|^{2},
$$

then

$$
\left|\sum_{j=1}^{n-1} z_{j}^{2}\right|+\frac{1}{2}\left|z_{n}\right|^{2}-1 \leq 0
$$


Thus

$$
\begin{aligned}
\sum_{j=1}^{n-1}\left|z_{j}\right|^{2} & =\sum_{j=1}^{n}\left|z_{j}\right|^{2}-\left|z_{n}\right|^{2}<\frac{1}{2}\left(1+\left|\sum_{j=1}^{n} z_{j}^{2}\right|^{2}\right)-\left|z_{n}\right|^{2} \\
& =\frac{1}{2}\left(1+\left|\sum_{j=1}^{n-1} z_{j}^{2}+z_{n}^{2}\right|^{2}\right)-\left|z_{n}\right|^{2} \\
& \leq \frac{1}{2}\left(1+\left(\left|\sum_{j=1}^{n-1} z_{j}^{2}\right|+\left|z_{n}\right|^{2}\right)^{2}\right)-\left|z_{n}\right|^{2} \\
& =\frac{1}{2}\left(1+\left|\sum_{j=1}^{n-1} z_{j}^{2}\right|^{2}\right)+\left|\sum_{j=1}^{n-1} z_{j}^{2}\right|\left|z_{n}\right|^{2}+\frac{1}{2}\left|z_{n}\right|^{4}-\left|z_{n}\right|^{2} \\
& =\frac{1}{2}\left(1+\left|\sum_{j=1}^{n-1} z_{j}^{2}\right|^{2}\right)+\left|z_{n}\right|^{2}\left(\left|\sum_{j=1}^{n-1} z_{j}^{2}\right|+\frac{1}{2}\left|z_{n}\right|^{2}-1\right) \\
& \leq \frac{1}{2}\left(1+\left|\sum_{j=1}^{n-1} z_{j}^{2}\right|^{2}\right) \cdot
\end{aligned}
$$

This completes the proof of (6).

Reapplying (4) recursively we obtain $\left(z_{1}, z_{2}, \ldots, z_{m}\right) \in D_{m}$ for all $m=$ $1, \ldots, k-1$. This proves part (a).

To prove part (b), let us use the notation $u=z_{1}+i z_{2}, w=z_{1}-i z_{2}$. It is immediate that $u w=z_{1}^{2}+z_{2}^{2}$ and that $|u|^{2}+|w|^{2}=2\left(\left|z_{1}\right|^{2}+\left|z_{2}\right|^{2}\right)$. Thus $\left(z_{1}, z_{2}\right) \in D_{2}$ if and only if

$$
\frac{1}{2}\left(|u|^{2}+|w|^{2}\right)<\frac{1}{2}\left(1+|u w|^{2}\right)<1 .
$$

The left-hand inequality corresponds to $\left(1-|u|^{2}\right)\left(1-|w|^{2}\right)>0$ and the righthand one to $|u w|<1$. Both inequalities are satisfied precisely when $|u|<1$ and $|w|<1$, proving (b).

For part (c), we use the same notation as in part (b). In addition, let $v=z_{3}$. Then $\left(z_{1}, z_{2}, z_{3}\right) \in D_{3}$ if and only if

$$
\frac{1}{2}\left(|u|^{2}+|w|^{2}\right)+|v|^{2}<\frac{1}{2}\left(1+\left|u w+v^{2}\right|^{2}\right)<1 .
$$

To get the result, we shall study only the left-hand inequality, which we rewrite in the form

$$
1-|u|^{2}-|w|^{2}+\left|u w+v^{2}\right|^{2}-2|v|^{2}>0 .
$$

Notice that $\left|u w+v^{2}\right|^{2}=|u|^{2}|w|^{2}+2 \operatorname{Re}\left(u w \bar{v}^{2}\right)+|v|^{4}$. But $2 \operatorname{Re}\left(u w \bar{v}^{2}\right) \leq$ $2|u||w||v|^{2} \leq\left(|u|^{2}+|w|^{2}\right)|v|^{2}$. From (7), then, we obtain that

$$
1-|u|^{2}-|w|^{2}+|u|^{2}|w|^{2}+\left(|u|^{2}+|w|^{2}\right)|v|^{2}+|v|^{4}-2|v|^{2}>0 \text {. }
$$

But the left-hand side factors as $\left(1-|u|^{2}-|v|^{2}\right)\left(1-|w|^{2}+|v|^{2}\right)$. This proves that $|u|^{2}+|v|^{2}$ and $|w|^{2}+|v|^{2}$ are never equal to 1 . From the connectedness 
of $D_{3}$, it follows that these quantities must both be always greater than 1 or both always less than 1 . Since they both vanish at the origin, they must both be always less than 1 . This proves part (c).

We are now ready to prove Theorem 2 .

Proof. For each type of Cartan classical domain it will suffice to find a holomorphic function $f: D \rightarrow \Delta$ and $x \in \partial D$ such that $H_{0}(x, \bar{x})=r_{D}^{2}$ and $\nabla_{0}(f) x=1$.

We shall first consider the case when $D$ is of type $\mathrm{I}_{m, n}$ or $\mathrm{II}_{n}$. Let $f$ be the function with domain $D$ defined by $f(Z)=z_{11}$, for all $Z=\left(\left(z_{i j}\right)\right) \in D$. This makes sense, since we may consider the free variables to be all entries $z_{i j}$ for case $\mathrm{I}_{m, n}$ and $z_{11}, z_{12}, \ldots, z_{1 n}, z_{22}, \ldots, z_{2 n}, z_{33}, \ldots, z_{3 n}, \ldots, z_{n n}$, for case $\mathrm{II}_{n}$. Then $f$ is holomorphic in $D$, and by Lemma 2 has image contained in $\Delta$. Let $\Xi$ be as in (1). Then $\Xi \in \partial D, \nabla_{0}(f)=\Xi$. Consequently $H_{0}(\Xi, \bar{\Xi})=r_{D}^{2}$ and $\nabla_{0}(f) * \Xi=1$.

Now let $D$ be of type III $_{n}$. Let $f$ be the function with domain $D$ defined by $f(Z)=z_{12}$, for all $Z=\left(\left(z_{i j}\right)\right) \in D$. We may consider the free variables to be $z_{12}, \ldots, z_{1 n}, z_{23}, \ldots, z_{2 n}, z_{34}, \ldots, z_{3 n}, \ldots, z_{n-1, n}$. As in the previous cases, we see that $f$ is holomorphic in $D$, and has image contained in $\Delta$. Now let $\Xi$ be as in (2). Then $\nabla_{0}(f)=\Xi$, and $\Xi \in \partial D$. Thus $\nabla_{0}(f) * \Xi=1$ and $H_{0}(\Xi, \bar{\Xi})=r_{D}^{2}$.

Now let $D$ be of type $\mathrm{IV}_{n}$. Define $f: D \rightarrow \mathbb{C}$ by $f\left(z_{1}, \ldots, z_{n}\right)=z_{1}+i z_{2}$. Then $f$ is holomorphic. By Lemma $3(\mathrm{a}, \mathrm{b})$ the function $f$ maps $D$ into the unit disk. Furthermore $\nabla(f)=(1, i, 0, \ldots, 0)$.

Thus the point $\xi=\left(\frac{1}{2},-\frac{i}{2}, 0, \ldots, 0\right)$ of $\partial D$ satisfies the conditions $H_{0}(\xi, \bar{\xi})=r_{D}^{2}$ and $\nabla_{0}(f)=\xi=1$.

This completes the proof.

As an immediate consequence of Theorem 2 we obtain

Corollary 1. If $D$ is a Cartan classical domain then

$$
c_{D}= \begin{cases}\sqrt{2 /(m+n)}, & \text { if } D \text { is of iype } \mathrm{I}_{m, n}, \\ \sqrt{2 /(n+1)}, & \text { if } D \text { is of type } \mathrm{II}_{n}, \\ 1 / \sqrt{n-1}, & \text { if } D \text { is of type } \mathrm{III}_{n}, \\ \sqrt{2 / n}, & \text { if } D \text { is of type } \mathrm{IV}_{n} .\end{cases}
$$

Theorem 3. If $D$ is a bounded symmetric domain with no exceptional factors, then $c_{D}=\max _{j} c_{D_{j}}$, where $D_{1}, \ldots, D_{k}$ are the irreducible factors of $D$. The constants $c_{D_{j}}$ are given in Corollary 1.

Proof. Without loss of generality, we may assume that $D=D_{1} \times \cdots \times D_{k}$, where each $D_{j}$ is in standard form. Since the Bergman metric $H_{z}$ of a product space is the direct sum of the Bergman metrics $H_{z_{j}}^{j}$ of the individual factors, and the smallest norm of any point on the boundary occurs when all but one of the coordinates is 0 , we have

$$
r_{D}^{2}=\min _{x \in \partial D} H_{0}(x, \bar{x})=\min _{j=1, \ldots, k} \min _{x_{j} \in \partial D_{j}} H_{0}^{j}\left(x_{j}, \overline{x_{j}}\right)=\min _{j=1, \ldots, k} r_{D_{j}}^{2} .
$$

Thus $c_{D} \leq \max _{j=1, \ldots, k} c_{D_{j}}$. 
Assume that this maximum is attained at $D_{j}$. Then let $f: D_{j} \rightarrow \Delta$ be a holomorphic function such that $\nabla_{0}(f) \xi=1$, where $\xi \in \partial D_{j}$ and the minimum of $H_{0}^{j}\left(x_{j}, \overline{x_{j}}\right)$ for $x_{j} \in \partial D_{j}$ is attained at $\xi$. The existence of $f$ is guaranteed by Theorem 2 . Define the holomorphic function $g: D \rightarrow \Delta$ by $g\left(z_{1}, \ldots, z_{k}\right)=$ $f\left(z_{j}\right)$. Then $\nabla_{0}(g)(0, \ldots, 0, \xi, 0, \ldots, 0)=1$, where $\xi$ occupies the $j$ th place, and $(0, \ldots, 0, \xi, 0, \ldots, 0)$ is a point of $\partial D$ where the minimum over all $x \in \partial D$ of $H_{0}(x, \bar{x})$ is attained. Thus $c_{D}=c_{D_{j}}$.

\section{SOME SCHWARZ-TYPE LEMMAS}

The following is a variation of the equality case in Schwarz's Lemma for a wide class of balanced (possibly unbounded) domains in $\mathbb{C}^{n}$. It will be used later in the proof of Theorem 6.

Lemma 4. Let $D_{1}$ and $D_{2}$ be balanced domains. Let $h: D_{1} \times D_{2} \rightarrow \Delta$ be $a$ holomorphic function such that $h(\mathbf{0})=0,\left(\partial h(\mathbf{0}) / \partial z_{1}\right) x=1$ for some $x \in \partial D_{1}$, and $\partial h(\mathbf{0}) / \partial z_{2}=0$. Then $h(z x, w)=z$ for all $w \in D_{2}$ and $z \in \Delta$.

Proof. Let $z_{2} \in D_{2}$ be arbitrary. Then define $g: \Delta \rightarrow \Delta$ by $g(z)=h\left(z x, z z_{2}\right)$. Note that $g(0)=0$, and

$$
g^{\prime}(0)=\frac{\partial h}{\partial z_{1}}(\mathbf{0}) x+\frac{\partial h}{\partial z_{2}}(\mathbf{0}) z_{2}=1 .
$$

Thus $g(z)=z$, by Schwartz's Lemma. This means that for all $z_{2} \in D_{2}$ and $z \in \Delta$, we have $h\left(z x, z z_{2}\right)=z$. Fixing $z \in \Delta, z \neq 0$, the set of all $w$ of the form $z z_{2}$ with $z_{2}$ in $D_{2}$ is open in $D_{2}$. Thus $h(z x, w)=z$ on an open set of $D_{2}$ and hence on all of $D_{2}$. So for all $w \in D_{2}$ and all $z \in \Delta, z \neq 0$, we have $h(z x, w)=z$. By continuity, then, $h(z x, w)=z$, for all $(z, w) \in \Delta \times D_{2}$.

As a special case of Lemma 4 with $D_{1}=\Delta$ we obtain

Lemma 5. Let $D$ be a balanced domain in $\mathbb{C}^{n}$. Let $h: \Delta \times D \rightarrow \Delta$ be a holomorphic function such that $h(\mathbf{0})=0, \partial h(\mathbf{0}) / \partial z_{1}=1$ and $\partial h(\mathbf{0}) / \partial z_{2}=0$. Then $h\left(z_{1}, z_{2}\right)=z_{1}$, for all $\left(z_{1}, z_{2}\right) \in \Delta \times D$.

It is not possible to find a formulation of the Schwarz Lemma from the $n$-ball to the disk as strong as that of Lemma 5 when $n>1$. The following result is a version of the Schwarz-Pick Lemma for the unit ball of $\mathbb{C}^{n}$.

Lemma 6. Let $f: B_{n} \rightarrow \Delta$ be holomorphic. Let $\xi=\left(0, \ldots, 0, \xi_{k+1}, \ldots, \xi_{n}\right) \in$ $B_{n}$, for $1 \leq k \leq n$. Then

$$
\left\|\left(\frac{\partial f}{\partial z_{1}}(\xi), \ldots, \frac{\partial f}{\partial z_{k}}(\xi)\right)\right\|\left(1-\|\xi\|^{2}\right)^{1 / 2} \leq 1-|f(\xi)|^{2} .
$$

Let $\xi_{j}=r \overline{\partial f(\xi) / \partial z_{j}}, j=1, \ldots, k$, where $r$ is the constant defined by the relation

$$
\left\|\left(\frac{\partial f}{\partial z_{1}}(\xi), \ldots, \frac{\partial f}{\partial z_{k}}(\xi)\right)\right\| r=\left(1-\|\xi\|^{2}\right)^{1 / 2} .
$$

Then equality in (8) holds if and only if the function

$$
g(z)=f\left(\xi_{1} z, \ldots, \xi_{k} z, \xi_{k+1}, \ldots, \xi_{n}\right),
$$


for $z \in \Delta$, is a (conformal) automorphism of the unit disk.

Proof. The points $\xi_{j}, j=1, \ldots, k$, were defined so that $\left(\xi_{1}, \ldots, \xi_{n}\right) \in \partial B_{n}$. So the function $g: \Delta \rightarrow \Delta$ given by $g(z)=f\left(\xi_{1} z, \ldots, \xi_{k} z, \xi_{k+1}, \ldots, \xi_{n}\right)$ is well-defined. Schwarz-Pick's Lemma applied to the analytic function $g$ yields

$$
\left|g^{\prime}(z)\right| \leq\left(1-|g(z)|^{2}\right) /\left(1-|z|^{2}\right) \text {. }
$$

In particular, for $z=0$ we obtain

$$
\left|\sum_{j=1}^{k} \frac{\partial f}{\partial z_{j}}(\xi) \xi_{j}\right| \leq 1-|f(\xi)|^{2} .
$$

But the left-hand side of (9) is given by

$$
r \sum_{j=1}^{k}\left|\frac{\partial f}{\partial z_{j}}(\xi)\right|^{2}=\left\|\left(\frac{\partial f}{\partial z_{1}}(\xi), \ldots, \frac{\partial f}{\partial z_{k}}(\xi)\right)\right\|\left(1-\|\xi\|^{2}\right)^{1 / 2},
$$

proving the inequality $(8)$.

Next assume that the equality holds in (8). Then we have that $\left|g^{\prime}(0)\right|=$ $1-|g(0)|^{2}$. By the equality case in the Schwarz-Pick Lemma, $g$ is an automorphism.

As a consequence of this result we get

Corollary 2. Let $f: B_{n} \rightarrow \Delta$ be holomorphic. Then for all $w \in B_{n}, w \neq 0$, we have

$$
\left\|\nabla_{w}(f)-\frac{1-s}{\|w\|^{2}}\left(\nabla_{w}(f) \cdot w\right) w\right\| \leq \frac{1-|f(w)|^{2}}{s},
$$

where $s=\left(1-\|w\|^{2}\right)^{1 / 2}$. For $w=0$ we obtain

$$
\left\|\nabla_{0}(f)\right\| \leq 1-|f(0)|^{2} \text {, }
$$

with equality holding in this latter case if and only if the function

$$
z \mapsto f\left(z \overline{\nabla_{0}(f)} /\left\|\nabla_{0}(f)\right\|\right)
$$

is an automorphism of the disk.

Proof. Applying Lemma 6 in the case $n=k$ yields the result for the case $w=0$. For arbitrary $w \neq 0$ in $B_{n}$, let $\varphi$ be the automorphism of $B_{n}$ sending 0 to $w$ given by

$$
\varphi(z)=\frac{w-(1-s) P(z)-s z}{1-z \cdot w}
$$

where $P(z)=(z \cdot w) w /\|w\|^{2}$ is the projection of $z$ onto the subspace generated by $w$. Then by $[R 1$, p. 26] we have that the matrix of partial derivatives of $\varphi$ at 0 is $-s(I-(1-s) P)$, where $I$ is the identity matrix. Set $g=f \circ \varphi$. Applying the previous part to $g$, we obtain $\left\|\nabla_{0}(g)\right\| \leq 1-|g(0)|^{2}$, which yields the result.

Theorem 4. Let $f: B_{n} \rightarrow \Delta$ be holomorphic such that $f(\mathbf{0})=0$ and $\partial f(0) / \partial z_{j}$ $=\delta_{j, k}$, for some fuxed $k \in\{1, \ldots, n\}$ and all $j \in\{1, \ldots, n\}$. Then $f$ is of the form

$$
f\left(z_{1}, \ldots, z_{n}\right)=z_{k}+\sum_{\substack{1 \leq l \leq j \leq m \\ l, j \neq k}} z_{l} z_{j} h_{l, j}\left(z_{1}, \ldots, z_{n}\right)
$$


where $h_{l, j}$ are holomorphic functions on $B_{n}$.

Proof. Without loss of generality, let us assume that $k=1$. From the hypothesis it follows that $f$ admits the representation

$$
f\left(z_{1}, \ldots, z_{n}\right)=z_{1}+z_{1} \sum_{j=1}^{n} z_{j} g_{j}\left(z_{1}\right)+\sum_{2 \leq l \leq j \leq m} z_{l} z_{j} h_{l, j}\left(z_{1}, \ldots, z_{m}\right),
$$

where each $g_{j}$ is analytic in the variable $z_{1}$ and each $h_{l, j}$ is a holomorphic function on $B_{n}$. We need to show that the functions $g_{j}$ are identically 0 .

Defining $g(z)=f(z, 0, \ldots, 0)=z+z^{2} g_{1}(z)$, we see that $g$ is an analytic function from $\Delta$ to itself such that $g(0)=0$ and $g^{\prime}(0)=1$. Thus from the Schwarz Lemma it follows that $g(z)=z$, and so $g_{1}$ must be identically 0 .

Fix $j \in\{2, \ldots, m\}$ and $z_{1} \in \Delta$, and let $r=\sqrt{1-\left|z_{1}\right|^{2}}$. Then

$$
\left|z_{1}\right|^{2}+r^{2}|z|^{2}=1-\left(1-\left|z_{1}\right|^{2}\right)\left(1-|z|^{2}\right)<1 \text {. }
$$

Thus for all $z \in \Delta$, we may define

$$
\begin{aligned}
h(z) & =f\left(z_{1}, 0, \ldots, 0, r z, 0, \ldots, 0\right) \\
& =z_{1}+r z_{1} z g_{j}\left(z_{1}\right)+r^{2} z^{2} h_{j, j}\left(z_{1}, 0, \ldots, 0, r z, 0, \ldots, 0\right),
\end{aligned}
$$

where $r z$ occupies the $j$ th coordinate. Since $h$ is an analytic function from $\Delta$ into itself, we have $\left|h^{\prime}(0)\right| \leq 1-|h(0)|^{2}$. Now $h(0)=z_{1}$ and $h^{\prime}(0)=$ $r z_{1} g_{j}\left(z_{1}\right)$. Thus $r\left|z_{1}\right|\left|g_{j}\left(z_{1}\right)\right| \leq 1-\left|z_{1}\right|^{2}=r^{2}$. Hence for all $z \in \Delta$ we obtain $|z|^{2}\left|g_{j}(z)\right|^{2} \leq 1-|z|^{2}$. So as $|z| \rightarrow 1, g_{j}(z)$ must approach 0 . By the maximum principle, $g_{j}$ must be identically 0 . This yields the desired representation of $f$.

Remark 3. The functions $f$ as in (10) are extremal. It should be noted, however, that it is not true that for arbitrary holomorphic functions $h_{l, j}$ on the ball the corresponding function $f$ necessarily maps $B_{n}$ into the unit disk. For instance, in the case $n=2$ we have the following result: If $h$ is a holomorphic function defined in the 2-ball such that $|h(z, w)| \leq 1 /(1+|z|)$ for all $(z, w) \in B_{2}$, then the function $f(z, w)=z+w^{2} h(z, w)$ maps $B_{2}$ into the unit disk, whence $f$ constitutes an extremal function in the 2-ball. Indeed if $|z|^{2}+|w|^{2}<1$ then

$$
\begin{aligned}
|f(z, w)| & \leq|z|+|w|^{2}|h(z, w)|<|z|+\left(1-|z|^{2}\right)|h(z, w)| \\
& \leq|z|+\left(1-|z|^{2}\right) \frac{1}{1+|z|}=1 .
\end{aligned}
$$

It would be interesting to find a condition in the opposite direction. A weak form of a converse is illustrated in the following result.

Proposition 2. If the function $f(z, w)=z+w^{2} h(z, w)$ maps $B_{2}$ into $\Delta$, then for all $z \in \Delta$ and $\rho \in(0,1)$ there exists $w \in \Delta$ such that $|w|^{2}=\rho\left(1-|z|^{2}\right)$ (so that $\left.(z, w) \in B_{2}\right)$ and $|h(z, w)| \leq 1 / \rho(1+|z|)$. In particular, for each $z \in \Delta$ we have that $\inf _{(z, w) \in B_{2}}|h(z, w)| \leq 1 /(1+|z|)$.

Proof. Fix $z \in \Delta$ and $\rho \in(0,1)$. If $h(z, w)$ is identically 0 as a function of $w$, the assertion is trivial. Otherwise the function $w \mapsto w^{2} h(z, w)$ is nonconstant analytic and maps 0 to 0 . Thus $w^{2} h(z, w)$ winds around the origin along any circle $\{|w|=r\}$ with $0<r<\sqrt{1-|z|^{2}}$. Choose $w$ so 
that $|w|=r$ and $\arg \left[w^{2} h(z, w)\right]=\arg (z)$. Thus $|z|+|w|^{2}|h(z, w)|=\mid z+$ $w^{2} h(z, w) \mid<1$, so $|h(z, w)|<(1-|z|) /|w|^{2}$. Set $r=\sqrt{\rho\left(1-|z|^{2}\right)}$. Then we obtain

$$
|h(z, w)|<\frac{1-|z|}{\rho\left(1-|z|^{2}\right)}=\frac{1}{\rho(1+|z|)} .
$$

\section{THE EXTREMAL FUNCTIONS}

In this section we provide a partial description of the extremal functions whose domain is a classical bounded symmetric domain. We are unable to present analogous results when there is an exceptional factor present, because we do not even have an estimate on the inner radius of the exceptional domains. First we give a technical result that will be used to reduce the problem to the study of a single irreducible factor.

Proposition 3. (i) Let $A$ be a positive definite matrix in $\mathscr{M}_{n}$, with $A(x, y)$ the associated positive definite bilinear form. Let $B$ be the positive definite matrix such that $B^{2}=A^{-1}$ and let $v \in \mathbb{C}^{n}$. Then

$$
\sup _{x \neq 0} \frac{|v \cdot x|}{A(x, \bar{x})^{1 / 2}}=\|B v\|
$$

and this supremum is a maximum.

(ii) Let $A_{j}(x, y), j=1, \ldots, k$, be positive definite bilinear forms of (possibly different) dimensions $n_{j}$ with associated matrices $A_{j}$, and let $v_{j} \in \mathbb{C}^{n_{j}}$. Let $A$ be the matrix constructed by putting the matrices $A_{j}$ along the main diagonal and zeros elsewhere. Let $v=\left(v_{1}, \ldots, v_{k}\right)$ be in $\mathbb{C}^{n}$, with $n=\sum_{j=1}^{k} n_{j}$. Let $S_{j}=\sup _{x_{j} \neq 0}\left|v_{j} \cdot x_{j}\right| / A_{j}\left(x_{j}, \overline{x_{j}}\right)^{1 / 2}$. Then

$$
\sup _{x \neq 0} \frac{|v \cdot x|}{A(x, \bar{x})^{1 / 2}}=\sqrt{\sum_{1 \leq j \leq k} S_{j}^{2}}=\left\|\left(S_{1}, \ldots, S_{k}\right)\right\| .
$$

Proof. First we notice that the supremum is a maximum because the quotient remains unchanged when any $x$ is replaced by a nonzero multiple of $x$. We can thus take the supremum over the set of all $x$ of norm 1 , but this is a compact set, so the supremum is attained. Let us prove (i).

If $A$ is the identity matrix (whence $B$ is also), then $A(x, y)=x \cdot \bar{y}$. In this case the result is true because $|v \cdot x| /\|x\|$ reaches its maximum $\|v\|$ at $x=v$. In the general case, observing that $B$ is selfadjoint, we have $A(x, y)=$ $(A x) \cdot \bar{y}=\left(B^{-2} x\right) \cdot \bar{y}=\left(B^{-1} x\right) \cdot\left(B^{-1} \bar{y}\right)$, so that $A(x, \bar{x})=\left\|B^{-1} x\right\|^{2}$. Thus

$$
\sup _{x \neq 0} \frac{|v \cdot x|}{A(x, \bar{x})^{1 / 2}}=\sup _{x \neq 0} \frac{|v \cdot x|}{\left\|B^{-1} x\right\|} \text {. }
$$

Since $B$ is nonsingular, without loss of generality, we can replace each $x$ by $B x$ so that the right-hand side of the previous equality is $\sup _{x \neq 0}|v \cdot B x| /\|x\|=$ $\sup _{x \neq 0}|B v \cdot x| /\|x\|=\|B v\|$, by the identity case.

Next note that (ii) follows from (i) since $B^{2}=A^{-1}$ if $B$ is the matrix made up by putting the $B_{j}$ along the main diagonal and 0 elsewhere, where 
$B_{j}^{2}=A_{j}^{-1}$. Thus

$$
B v=\left(\begin{array}{c}
B_{1} v_{1} \\
B_{2} v_{2} \\
\vdots \\
B_{k} v_{k}
\end{array}\right)
$$

and so

$$
\|B v\|=\sqrt{\sum_{1 \leq j \leq k}\left\|B_{j} v_{j}\right\|^{2}} .
$$

Applying part (i) we get the result.

For convenience, let us use the following notation: if $f: D=D_{1} \times D_{2} \times$ $\cdots \times D_{k} \rightarrow \mathbb{C}$ is holomorphic, then for $j=1, \ldots, k$, by $\partial f(z) / \partial z_{j}$ we mean the gradient of $f$ taken with respect to the variables which are in $D_{j}$ only. So if $D_{j}$ is a domain in $\mathbb{C}^{t}, \partial f(z) / \partial z_{j}$ is in $\mathbb{C}^{t}$. Thus $\nabla(f)=$ $\left(\partial f / \partial z_{1}, \ldots, \partial f / \partial z_{k}\right)$. Fixing the point $z=\left(z_{1}, \ldots, z_{k}\right) \in D=D_{1} \times D_{2} \times$ $\cdots \times D_{k}$, let $f_{j}: D_{j} \rightarrow \mathbb{C}$ be defined by

$$
f_{j}(w)=f\left(z_{1}, \ldots, z_{j-1}, w, z_{j+1}, \ldots, z_{k}\right) .
$$

Note that $\nabla_{z_{j}}\left(f_{j}\right)=\partial f\left(z_{j}\right) / \partial z_{j}$. The Bergman metric on $D$ is given by $H_{z}(u, \bar{v})=\sum_{j=1}^{k} H_{z_{j}}^{j}\left(u_{j}, \bar{v}_{j}\right)$, where the subscript and superscript $j$ refer to the corresponding component $D_{j}$. By Proposition 3(ii), we get the following

Theorem 5. With the notation given above, let $D=D_{1} \times D_{2} \times \cdots \times D_{k}$, where each $D_{j}$ is a bounded symmetric domain. Let $f: D \rightarrow \Delta$ be holomorphic. Then

$$
\beta_{f}\left(z_{1}, \ldots, z_{k}\right)=\|\left(\beta_{f_{1}}\left(z_{1}\right), \ldots, \beta_{f_{k}}\left(z_{k}\right) \|,\right.
$$

for all $\left(z_{1}, \ldots, z_{k}\right) \in D$.

We can use this result to study extremal functions. We recall that in the definition of $\beta_{f}(z)$, the supremum is actually a maximum. In addition, since multiplying $x$ by a complex constant of modulus 1 leaves the quotient unchanged, we can find an $x$ so that

$$
\beta_{f}(z)=\nabla_{z}(f) x /\left[H_{z}(x, \bar{x})\right]^{1 / 2} \text {. }
$$

Now let $D_{j}$ be a classical domain in standard form, $j=1, \ldots, k$. Assume that $D=D_{1} \times D_{2} \times \cdots \times D_{k}$ and $f: D \rightarrow \Delta$ is extremal. Assume further that $\beta_{f}(0)=c_{D}$. For each $j=1, \ldots, k$, there exists $x_{j} \in \partial D_{j}$, such that $\beta_{f_{j}}(0)=\left(\partial f(0) / \partial z_{j}\right) x_{j} /\left[H_{0}^{j}\left(x_{j}, \overline{x_{j}}\right)\right]^{1 / 2}$. Define $g: \Delta \rightarrow \Delta$ by $g(z)=$ $f\left(z x_{1}, \ldots, z x_{k}\right)$. Then

$$
\begin{aligned}
g^{\prime}(0) & =\nabla_{0}(f)\left(x_{1}, \ldots, x_{k}\right)=\sum_{j=1}^{k} \frac{\partial f}{\partial z_{j}}(0) x_{j} \\
& =\sum_{j=1}^{k} H_{0}^{j}\left(x_{j}, \overline{x_{j}}\right)^{1 / 2} \beta_{f_{j}}(0),
\end{aligned}
$$

which is a real number. By the Schwarz-Pick Lemma, we also know that $g^{\prime}(0) \leq$ 1 . 
Let $\mu=\min _{j} H_{0}^{j}\left(x_{j}, \overline{x_{j}}\right)^{1 / 2}$, so $\mu \geq r_{D}$. Then we have $\mu \sum_{j=1}^{k} \beta_{f_{j}}(0) \leq$ $g^{\prime}(0) \leq 1$. Now

$$
\beta_{f}(0)=\left\|\left(\beta_{f_{1}}(0), \ldots, \beta_{f_{k}}(0)\right)\right\| \leq \sum_{j=1}^{k} \beta_{f_{j}}(0) \leq \frac{1}{\mu} \leq \frac{1}{r_{D}}=c_{D}=\beta_{f}(0) .
$$

Thus all the inequalities are equalities. But the first inequality of (11) can be an equality only if all but one of the components of $\left(\beta_{f_{1}}(0), \ldots, \beta_{f_{k}}(0)\right)$ is zero. Thus we see that for some $m \in\{1, \ldots, k\}$ we have $\beta_{f_{j}}(0)=0$ for all $j \neq m$ and $\beta_{f_{m}}(0)=c_{D}$. In particular this means that $c_{D}=c_{D_{m}}$ and $f_{m}$ is extremal on $D_{m}$. The fact that all inequalities are equalities also means that $g^{\prime}(0)=1$, $\mu=H_{0}^{m}\left(x_{m}, \overline{x_{m}}\right)^{1 / 2}$, and $\mu \beta_{f_{m}}(0)=1$. In particular, by the Schwarz-Pick Lemma, $g(z)=z$, and $f(0)=0$.

For all $j \neq m$, we have $\beta_{f_{j}}(0)=0$, and so we also have $\partial f(0) / \partial z_{j}=0$. Using the fact that $f_{m}$ is extremal on $D_{m}$, we obtain that $\left(\partial f(0) / \partial z_{m}\right) x_{m}=1$. Letting $D_{m}$ be the domain $D_{1}$ and the product of the other factors of $D$ the domain $D_{2}$ of Lemma 4 we see that

$$
f\left(z_{1}, \ldots, z_{m-1}, z x_{m}, z_{m+1}, \ldots, z_{k}\right)=z, \quad \text { for all } z_{j} \in D_{j}, j \neq m .
$$

Now instead assume $\beta_{f}(w)=c_{D}$, for some $w=\left(w_{1}, \ldots, w_{k}\right) \in D$. For each $j \in\{1, \ldots, k\}$ let $S_{j}$ be an automorphism of $D_{j}$ such that $S_{j}(0)=w_{j}$. Setting $\Psi=S_{1} \times \cdots \times S_{k}$ and $F=f \circ \Psi$, we obtain that $F: D \rightarrow \Delta$ is holomorphic and $\beta_{F}(0)=c_{D}$. Thus for some $m \in\{1, \ldots, k\}$ there exists $x_{m} \in \partial D_{m}$ such that $F\left(z_{1}, \ldots, z_{k}\right)=z$, where $z_{m}=z x_{m}$ and $z_{j} \in D_{j}$ is arbitrary for $j \neq m$. Thus $f\left(S_{1}\left(z_{1}\right), \ldots, S_{m}\left(z x_{m}\right), \ldots, S_{k}\left(z_{k}\right)\right)=z$. Since $S_{j}\left(z_{j}\right)$ is an arbitrary element of $D_{j}$ for $j \neq m$, we get

Theorem 6. Let $D=D_{1} \times D_{2} \times \cdots \times D_{k}$ be a classical domain in standard form, and assume that $f: D \rightarrow \Delta$ is holomorphic and such that $\beta_{f}(w)=c_{D}$, for some $w \in D$. Then for some $m \in\{1, \ldots, k\}, c_{D}=c_{D_{m}}$ and there exist $x_{m} \in \partial D_{m}$ and an automorphism $T$ of $D_{m}$ such that

$$
f\left(z_{1}, \ldots, z_{m-1}, T\left(z x_{m}\right), z_{m+1}, \ldots, z_{k}\right)=z,
$$

for all $z \in \Delta$, and $z_{j} \in D_{j}$ for all $j \neq m$.

Notice that in the above theorem, if any of the domains $D_{j}$ is the unit disk, then $c_{D}=1$ and $D_{m}$ must be equal to $\Delta$. In this case $z x_{m}$ is just an arbitrary element of $D_{m}$. Setting $z_{m}=T\left(z x_{m}\right)$ and $S(w)=\bar{x}_{m} T^{-1}(w)$, so that $S\left(z_{m}\right)=z$, we then get

Corollary 3. Let $D=D_{1} \times D_{2} \times \cdots \times D_{k}$ be a classical domain in standard form where at least one of the $D_{j}$ is $\Delta$. Assume that $f: D \rightarrow \Delta$ is holomorphic and such that $\beta_{f}(w)=c_{D}$, for some $w \in D$. Then for some $m \in\{1, \ldots, k\}$ with $D_{m}=\Delta, c_{D}=c_{D_{m}}(=1)$, and there exists a conformal automorphism $S$ of $\Delta$ such that

$$
f\left(z_{1}, \ldots, z_{k}\right)=S\left(z_{m}\right) .
$$

In order to complete the study of extremal functions from a classical domain $D$ having $\Delta$ as an irreducible factor into the unit disk, we need to look at functions $f: D \rightarrow \Delta$ with $c_{D}=\beta_{f}$ but with $\beta_{f}(w)<c_{D}$, for all $w \in D$. We get 
Theorem 7. Let $D$ be a bounded symmetric domain and $f: D \rightarrow \Delta$ a holomorphic function with $\beta_{f}=c_{D}$. For each $w_{0} \in D$, there exists a sequence $\left\{T_{n}\right\}_{n \in \mathbf{N}}$ of automorphisms of $D$ such that the sequence $\left\{f \circ T_{n}\right\}$ converges uniformly on compact subsets to a holomorphic function $F: D \rightarrow \Delta$ such that $\beta_{F}\left(w_{0}\right)=c_{D}$.

Proof. First note that there is a sequence $\left\{w^{(t)}\right\}_{t \in \mathbb{N}}$ in $D$ such that

$$
\lim _{t \rightarrow \infty} \beta_{f}\left(w^{(t)}\right)=c_{D}
$$

Fix $w_{0} \in D$. For each $t \in \mathbb{N}$ there exists an automorphism $S^{(t)}$ of $D$ such that $S^{(t)}\left(w_{0}\right)=w^{(t)}$. Thus $\lim _{t \rightarrow \infty} \beta_{f \circ S^{(t)}}\left(w_{0}\right)=c_{D}$. Since the sequence $\left\{f \circ S^{(t)}\right\}$ is uniformly bounded, it is a normal family (cf. [R1, p. 5]). Thus it has a subsequence $\left\{f \circ S^{\left(t_{n}\right)}\right\}_{n \in \mathbb{N}}$ which converges uniformly on compact subsets of $D$ to some holomorphic function $F$, whose image must be contained in $\Delta$. Letting $T_{n}=S^{\left(t_{n}\right)}$, we obtain $F=\lim _{n \rightarrow \infty} f \circ T_{n}$. It follows that $\beta_{F}\left(w_{0}\right)=$ $\lim _{n \rightarrow \infty} \beta_{f \circ T_{n}}\left(w_{0}\right)=c_{D}$.

Specializing to the case when $D$ is a classical domain with the unit disk as a factor, we obtain

Corollary 4. Let $D$ be a classical domain which has the unit disk as a factor, and $f: D \rightarrow \Delta$ holomorphic with $\beta_{f}=c_{D}$. Then there exist a sequence $\left\{\Phi_{n}\right\}_{n \in \mathbb{N}}$ of automorphisms of $D$ and an integer $m \in\{1, \ldots, k\}$ such that $D_{m}=\Delta$ and

$$
\lim _{n \rightarrow \infty} f \circ \Phi_{n}\left(z_{1}, \ldots, z_{k}\right)=z_{m} \text {, }
$$

for all $\left(z_{1}, \ldots, z_{k}\right) \in D$.

Proof. First apply Theorem 7 with $w_{0}=0$. By Theorem 6, the function $F$ that we obtain must be of the form

$$
F\left(z_{1}, \ldots, z_{k}\right)=\lambda z_{m}
$$

for some unimodular constant $\lambda$ and some $m \in\{1, \ldots, k\}$ for which $D_{m}=\Delta$. Setting $\Phi_{n}\left(z_{1}, \ldots, z_{k}\right)=T_{n}\left(z_{1}, \ldots, z_{m-1}, \bar{\lambda} z_{m}, z_{m+1}, \ldots, z_{k}\right)$, we obtain the assertion.

For the case of a bounded symmetric domain $D$ for which the unit disk is not an irreducible factor we are going to show that the class of extremal functions is very large indeed. In fact, we shall see that it contains an infinite direct product of copies of the space of holomorphic functions from $D$ to $\Delta$.

First we prove the following lemma.

Lemma 7. Let $D$ be a Cartan classical domain other than the unit disk. Then we can find two free complex variables, $u$ and $v$, of $D$ such that $|u|^{2}+|v|^{2}<1$, and such that the projection of $D$ onto the $u$-variable is an extremal function.

Proof. First consider $D$ of type I, II, or III. Since $D \neq \Delta$, the following restrictions hold:

(1) $m \geq 2$ for $D$ of type $\mathrm{I}_{m, n}$,

(2) $n \geq 2$, for $D$ of type $\mathrm{II}_{n}$,

(3) $n \geq 3$, for $D$ of type III $_{n}$.

This implies that the first row of any element $Z=\left(\left(z_{i j}\right)\right)$ of $D$ must have at least two (free) entries $u$ and $v$. We let $(u, v)=\left(z_{11}, z_{12}\right)$ for cases $\mathbf{I}_{m, n}$ and $\mathrm{II}_{n}$, and $(u, v)=\left(z_{12}, z_{13}\right)$, for case $\mathrm{III}_{n}$. By Lemma 1 the rows of $Z$ have norm less than one. Thus $|u|^{2}+|v|^{2}<1$. 
Now let $p: D \rightarrow \Delta$ be the function defined by $p(Z)=u$. By the proof of Theorem 2 it follows that $p$ is extremal and $c_{D}=\beta_{p}(0)$.

Now let $D$ be a Cartan classic domain of type $\mathrm{IV}_{n}$ for $n \geq 3$. Then we may take the first three free variables of $D$ as $u=z_{1}+i z_{2}, w=z_{1}-i z_{2}$, $v=z_{3}$. Moreover the function $p: D \rightarrow \Delta$ defined by $p\left(z_{1}, \ldots, z_{n}\right)=u$ is extremal by the proof of Theorem 2. By Lemma 3(a, c) we have $|u|^{2}+|v|^{2}=$ $\left|z_{1}+i z_{2}\right|^{2}+\left|z_{3}\right|^{2}<1$, for all $\left(z_{1}, z_{2}, z_{3}, \ldots, z_{n}\right) \in D$.

This completes the proof.

Let $D=D_{1} \times \cdots \times D_{k}$ be a classical domain in $\mathbb{C}^{t+2}$ in standard form, where each $D_{j}$ is irreducible and distinct from the unit disk. Let $m \in\{1, \ldots, k\}$ be chosen so that $c_{D}=c_{D_{m}}$. By rearranging the factors of $D$ we may assume that $m=1$.

Applying Lemma 7 to $D_{1}$, let $u$ and $v$ be the free variables of $D_{1}$ such that $|u|^{2}+|v|^{2}<1$ (recall that $u$ and $v$ are the first two coordinates in $D_{1}$ ) and such that the projection $p$ of $D_{1}$ onto the first coordinate is an extremal function and $\beta_{p}(0)=c_{D_{1}}$.

Since $c_{D}=c_{D_{1}}$ it follows that the projection $P$ of $D$ onto the first coordinate is also extremal.

Following an idea due to Rudin (cf. [R1, p. 164]) set

$$
c_{1}=\frac{1}{2}, \quad c_{n}=\frac{1 \cdot 3 \cdot 5 \cdots(2 n-3)}{2^{n} n !}, \quad \text { for } n \geq 2,
$$

so that $\sum_{1}^{\infty} c_{n} t^{n}=1-\sqrt{1-t}$. Let $\left\{g_{j}\right\}_{n \in \mathbb{N}}$ be a sequence of holomorphic functions from $D$ into $\Delta$, that is, $g_{j} \in H^{\infty}(D, \Delta)$ for all $j \in \mathbb{N}$. Then the new function

$$
f\left(u, v, z_{1}, \ldots, z_{t}\right)=u+\sum_{j=1}^{\infty} c_{j} g_{j}\left(u, v, z_{1}, \ldots, z_{t}\right) v^{2 j}
$$

is holomorphic on $D$ and has image contained in $\Delta$, since

$$
\left|f\left(u, v, z_{1}, \ldots, z_{t}\right)\right|<|u|+\sum_{j=1}^{\infty} c_{j}|v|^{2 j}=|u|+1-\sqrt{1-|v|^{2}}<1 .
$$

Observe that the gradient of $f$ at 0 coincides with the gradient of $P$ at 0 . Thus $\beta_{f}(0)=\beta_{P}(0)=c_{D}$. Hence $f$ is extremal. We have thus proved

Theorem 8. Let $D$ be a classical domain in standard form which does not have $\Delta$ as a factor. Let $\left\{g_{j}\right\}_{j \in \mathbb{N}} \in \prod_{j=1}^{\infty} H^{\infty}(D, \Delta)$. Then the function $f$ defined by (12) is extremal; in fact $\beta_{f}(0)=c_{D}$.

\section{APPENDIX. THE EXCEPTIONAL DOMAINS}

We now provide a modified version of the description of the exceptional domains given by Ise [I1].

Let $\mathbb{H}$ be the space of the complex quaternions, that is, the four-dimensional algebra with $\mathbb{C}$-basis $1, i, j, k$, and with multiplication given by the relations $i j=-j i=k, i^{2}=j^{2}=-1$. On $\mathbb{H}$ define the map $\alpha \mapsto \hat{\alpha}$ as the $\mathbb{C}$ linear transformation sending 1 to itself and $i, j, k$ to their negatives. Give $\mathbf{K}=\mathbb{H} \oplus \mathbb{H} e$ (for a symbol $e$ ) the nonassociative Cayley multiplication by 
adding the following rules: for $\alpha, \beta \in \mathbb{H}, \alpha(\beta e)=(\beta \alpha) e,(\beta e) \alpha=(\beta \hat{\alpha}) e$, $(\alpha e)(\beta e)=-\hat{\beta} \alpha$. We fix the $\mathbb{C}$-basis $c_{0}=1, c_{1}=i, c_{2}=j, c_{3}=k, c_{4}=e$, $c_{5}=i e, c_{6}=j e, c_{7}=k e$.

We can extend the map - to $\mathrm{K}$ by letting $\widehat{\alpha e}=-\alpha e$ for any $\alpha \in \mathbb{H}$. In addition, we have the map $x \mapsto \bar{x}$ which sends each of the coefficients of the basis elements to its complex conjugate. Thus $\mathbf{K}$ turns out to be a nonassociative eight-dimensional algebra over $\mathbb{C}$. For any $y \in \mathbf{K}$, consider the linear map $B_{y}: \mathbf{K} \rightarrow \mathbf{K}$ given by $B_{y}(u)=\widehat{y u}$. With respect to the above basis we may think of $B_{y}$ as an element of $\mathscr{M}_{8}$. For $w=(\bar{x}, y) \in \mathbf{K} \oplus \mathbf{K}$, let $A_{w} \in \mathscr{M}_{8}$ be the matrix $\left(\left(x_{j} y_{k}\right)\right)$. With respect to this notation, and letting I be the identity matrix in $\mathscr{M}_{8}$, we get a representation of the domain $E_{\mathrm{III}}$ in $\mathbb{C}^{16}$ as

$$
\begin{aligned}
D=\{w=(\bar{x}, y) \in \mathbf{K} \oplus \mathbf{K}: & \\
& \left.\|w\|^{2} \mathrm{I}-\left(\begin{array}{cc}
0 & A_{w} \\
A_{w}^{*} & 0
\end{array}\right)-\frac{1}{2}\left(\begin{array}{cc}
B_{y} B_{y}^{*} & -B_{y} B_{x} \\
-B_{x}^{*} B_{y}^{*} & B_{x}^{*} B_{x}
\end{array}\right)<\mathrm{I}\right\},
\end{aligned}
$$

where \|\| denotes the Euclidean norm.

Now we shall discuss the other exceptional domain, $D=E_{\mathrm{VII}}$. This is a domain in $\mathbb{C}^{27}$. We shall describe it as a subspace of a certain 27-dimensional Jordan algebra $\mathcal{J}$. If $X=\left(\left(x_{j k}\right)\right)$ is a matrix over $\mathbf{K}$, by $\widehat{X}$ we mean the matrix $\left(\left(\widehat{x_{j k}}\right)\right)$, by $X^{T}$ we mean the transpose of $X$.

We let $\mathcal{J}$ be the space of all matrices $X \in \mathscr{M}_{3}(\mathbf{K})$, such that $X=\widehat{X}^{T}$. The three elements above the main diagonal are arbitrary elements of $\mathbf{K}$ and determine the three below the diagonal, while the three elements on the diagonal satisfy $\widehat{x_{t t}}=x_{t t}$, and they hence must be complex multiples of the basis element $c_{0}$. Thus as a complex vector space, $\mathscr{J}$ is isomorphic to $\mathbf{K}^{3} \oplus \mathbb{C}^{3}$, and is thus 27-dimensional over $\mathbb{C}$.

The space $\mathscr{J}$ is not a subalgebra of $\mathscr{M}_{3}(\mathbf{K})$, however, but it is closed under the Jordan multiplication $X \circ Y=\frac{1}{2}(X Y+Y X)$. On $\mathcal{J}$ there is an inner product defined by $(X, Y)=\operatorname{Trace}(X \circ Y)$. There is also a cross product defined by

$$
\begin{aligned}
X \times Y= & X \circ Y-\frac{1}{2} \operatorname{Trace}(X) Y-\frac{1}{2} \operatorname{Trace}(Y) X \\
& +\frac{1}{2}\{\operatorname{Trace}(X) \operatorname{Trace}(Y)-(X, Y)\} \mathbf{I},
\end{aligned}
$$

where $I$ is the identity matrix in $\mathscr{M}_{3}(\mathbf{K})$.

Now we define $C_{X}: \mathcal{J} \rightarrow \mathscr{J}$ by $C_{X}(Y)=X \times Y$. Since this is a C-linear transformation, we shall consider $C_{X}$ as an element of $\mathscr{M}_{27}$. Similarly we define the matrix $A_{X}$ by setting $A_{X}(Y)=(\widehat{X}, Y) X$. With respect to this notation, and letting $I$ be the identity matrix in $\mathscr{M}_{27}$, we get a representation of the domain $E_{\mathrm{VII}}$ in $\mathbb{C}^{27}$ as

$$
D=\left\{X \in \mathscr{J}:\|X\|^{2} \mathrm{I}+A_{X}-4 C_{X} C_{X}^{*}<\mathrm{I}\right\} .
$$

Unfortunately, we do not know a good expression for $H_{0}$ in the exceptional cases. Even if we had an explicit formulation, however, it would probably still be difficult to find the inner radius of these exceptional domains, because it may be quite challenging to describe their boundaries. 


\section{FURTHER REMARKS AND OPEN QUESTIONS}

In the case $D=\Delta$, if $\beta_{f}\left(w_{0}\right)=c_{D}(=1)$, for some $w_{0} \in D$, then $f$ is an automorphism of $D$ and $f\left(w_{0}\right)=0$. Thus we have a precise description of the extremal functions which realize the value $c_{D}$ at some point of their domain. This precision was carried over to the case in which $D$ is a classical domain with $\Delta$ as a factor (Corollary 3 ). In [C2] there is also a precise characterization of the extremal functions $f$ on the unit disk whose Bloch constant is not attained inside $\Delta$. They are the functions of the form $f=g B$, where $g$ is a nonvanishing function of supremum norm 1 and $B$ is a Blaschke product whose zeros form a sequence $\left\{z_{n}\right\}$ satisfying the condition

$$
\limsup _{n \rightarrow \infty}\left|g\left(z_{n}\right)\right| \prod_{k \neq n}\left|\frac{z_{n}-z_{k}}{1-\overline{z_{n}} z_{k}}\right|=1 \text {. }
$$

But in the corresponding case for the polydisk, we only have Corollary 4, which yields little information concerning the extremal functions. For the case $D=\Delta$, the factorization of bounded analytic functions into a product $g B$, with $g$ nonvanishing and $B$ a Blaschke product, played a crucial role. Even in the case $D=\Delta^{n}$ for $n>1$ there are no similar tools which are as powerful. It would be interesting to try to describe the extremal functions $f$ with $\beta_{f}(w)<$ $c_{D}$ for all $w$ in the polydisk. For bounded symmetric domains which do not contain a disk factor the problem of describing all the extremal functions for which the Bloch constant is attained in the domain is already very difficult, as Theorem 8 shows.

Beside these general questions, there remains the major immediate problem of extending all our results to domains that contain an exceptional factor. If this is possible, it means that $r_{D}=1 / c_{D}$ for all bounded symmetric domains, so that there ought to be an intrinsic definition of $r_{D}$. The definition we give depends entirely on representing $D$ as a balanced domain. In addition, it would be interesting to study the same questions on arbitrary bounded homogeneous domains or for other classes of smooth functions.

\section{BIBLIOGRAPHY}

[C1] E. Cartan, Sur les domains bournés de l'espace de $n$ variable complexes, Abh. Math. Sem. Univ. Hamburg 11 (1935), 116-162.

[C2] F. Colonna, The Bloch constant of bounded analytic functions, J. London Math. Soc. (2) 36 (1987), 95-101.

[C3] - The Bloch constant of bounded harmonic mappings, Indiana Univ. Math. J. 38 (1989), 829-840.

[D1] D. Drucker, Exceptional Lie algebras and the structure of Hermitian symmetric spaces, Mem. Amer. Math. Soc. 208 (1978), 1-207.

[H1] K. T. Hahn, Holomorphic mappings of the hyperbolic space into the complex Euclidean space and the Bloch theorem, Canad. J. Math. 27 (1975), 446-458.

[H2] M. Heins, Selected topics in the classical theory of functions of a complex variable, Holt, Rinehart and Winston, New York, 1962.

[H3] S. Helgason, Differential geometry and symmetric spaces, Academic Press, San Diego, 1962.

[I1] M. Ise, Bounded symmetric domains of exceptional type, J. Fac. Sci. Univ. Tokyo Sect. IA Math. 23 (1976), 75-105.

[K1] S. Kobayashi, Hyperbolic manifolds and holomorphic mappings, Marcel Dekker, New York, 1970. 
[K2] M. Koecher, An elementary approach to bounded symmetric domains, Rice Univ., Houston, 1969.

[PS] I. I. Pyatetskii-Shapiro, Automorphic functions and the geometry of classical domains, Gordon and Breach, New York, 1969.

[R1] W. Rudin, Function theory in the unit ball of $\mathbb{C}^{n}$, Springer-Verlag, Berlin and New York, 1980.

[S1] C. L. Siegel, Analytic functions of several complex variables, Princeton Univ. Press, Princeton, NJ, 1950.

[T1] R. M. Timoney, Bloch functions in several complex variables. I, Bull. London Math. Soc. 12 (1980), 241-267.

[T2] $\frac{}{1-22}$. Bloch functions in several complex variables. II, J. Reine Angew. Math. 319 (1980),

Department of Mathematics, University of Maryland, College Park, Maryland 20742

E-mail address: jmc@amanda.umd.edu

Department of Mathematical Sciences, George Mason University, Fairfax, Virginia 22030

E-mail address: fcolonna@gmuvax.gmu.edu 